\title{
Development and internal validation of a diagnostic score for gastric linitis plastica
}

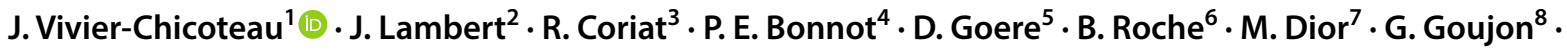 \\ S. Morgant ${ }^{3} \cdot$ M. Pocard ${ }^{9} \cdot$ O. Glehen ${ }^{4} \cdot$ T. Aparicio $^{1} \cdot$ Jean-Marc Gornet ${ }^{1}$
}

Received: 8 October 2019 / Accepted: 14 February 2020 / Published online: 26 February 2020

(c) The International Gastric Cancer Association and The Japanese Gastric Cancer Association 2020

\begin{abstract}
Background There is no consensual definition for gastric linitis plastica (GLP). We aim to construct a diagnostic score to distinguish this rare tumor from usual gastric adenocarcinomas.

Methods In this retrospective study, all patients who had gastrectomy for cancer between 2007 and 2017 in French tertiary centers were included. The outcome was a diagnosis of GLP based on pathological review of the surgical specimen. The diagnostic score was created by using variables that were most frequently associated with GLP using penalized logistic regression on multiply imputed datasets. We used discrimination measures to assess the performances of the score. Internal validation was performed using bootstrapping methods to correct for over-optimism.

Results 220 patients including 71 linitis plastica (female 49\%, median age 57 years) were analyzed. The six parameters retained in the diagnosis score were the presence of large folds and/or parietal thickening on at least one segment, pangastric infiltration and presence of gastric stenosis on the upper endoscopy, circumferential thickening on at least one segment and thickening of the third hyperechogenic layer on endoscopic ultrasound and the presence of signet ring cells on endoscopic biopsies. The area under the ROC curve (AUC) was 0.967 with a sensitivity of $94 \%$ [89.9-97.3] and a specificity of $88.7 \%$ [81.7-95.8] for a threshold of 2.75. After internal validation, the corrected AUC was 0.959.

Conclusion It is the first study validating a pre-therapeutic diagnostic score (Saint Louis linitis score) with an excellent ability to discriminate GLP from non-GLP adenocarcinomas. An external validation is necessary to confirm our data.
\end{abstract}

Keywords Linitis plastica · Diagnostic score · Gastrectomy

Electronic supplementary material The online version of this article (https://doi.org/10.1007/s10120-020-01051-x) contains supplementary material, which is available to authorized users.

Jean-Marc Gornet

jean-marc.gornet@aphp.fr

1 Service de Gastroentérologie, Hôpital Saint Louis, 1 Avenue Claude Vellefaux, 75010 Paris, France

2 Service de Biostatistique, Hôpital Saint-Louis, Paris, France

3 Service de Gastroentérologie, Hôpital Cochin, Paris, France

4 Service de Chirurgie Digestive, Centre Hospitalier Lyon-Sud, Lyon, France

5 Service de Chirurgie Digestive, Hôpital Saint-Louis, Paris, France

\section{Introduction}

Gastric adenocarcinoma (GA) is the fifth most common cancer in the world [1]. Despite medico-surgical progress, its prognosis remains poor, ranking third among the most fatal cancers $[1,2]$. There are various classifications of GA,

6 Service D'Anatomopathologie, Hôpital Saint Louis, Paris, France

7 Service de Gastroentérologie, Hôpital Louis Mourier, Colombes, France

8 Service de Gastroentérologie, Hôpital Bichat, Paris, France

9 Service de Chirurgie Digestive, Hôpital Lariboisière, Paris, France 
either purely histological $[3,4,5]$ or taking into account the macroscopic aspect [6], or the site of the tumor [7]. Among the different subtypes, gastric linitis plastica (GLP) represents a particular entity. It develops from the submucosa and is characterized macroscopically by a major segmental or diffuse thickening of the gastric wall and microscopically by the existence of poorly cohesive and/or signet ring cells, within an abundant fibrous stroma infiltrating all the tunics $[8,9]$. The terms of poorly cohesive and/or signet ring cell carcinoma (SRC) and GLP are often indiscriminately used leading to confusion in literature and difficulties to define the best therapeutic options for this subtype of gastric tumor. GLP appears to have specific characteristics such as younger age at diagnosis, female predominance, increased frequency of stages 3 and 4 and lymph node invasion, and significantly decreased overall survival due to higher frequency of R1 resection $[10,11]$. Despite these specific features, there is to date no clear definition of GLP. A recent consensus on the pathological definition and classification of poorly cohesive gastric carcinoma proposes that GA should be classified according to the WHO classification; the term GLP being reserved for the description of the macroscopic characteristics of the tumor [12]. According to those discrepancies, the gold standard for GLP diagnosis is currently based on histological examination of a surgical specimen [13, 14]. However in case of locally advanced or metastatic disease which represents the vast majority of the patients, surgery is rarely done. Thus, the diagnosis of GLP is mainly based on a simple set of arguments (clinical, endoscopic, scannographic, histological). In case of a planned surgery for localized GLP, the impact of preoperative chemotherapy remains uncertain and a total gastrectomy is needed even in case of peroperative impression of free margin. The development of a new diagnostic tool to make an early diagnosis of GLP remains challenging and may lead to better understanding and significant therapeutic advances in this field. The aim of this study is to construct a diagnostic score to discriminate GLP from others GA.

\section{Methods}

All patients who underwent a gastrectomy for gastric cancer between 2007 and 2017 in seven French tertiary centers were retrospectively identified either from a hospital database known as Programme de Médicalisation des Systèmes d'Information (PMSI) or from databases of the gastroenterology departments. All the files were reviewed by the same person (JVC) to minimize missing data and control concordance; collected data included information concerning demographic characteristics, case history, biological parameters, description of endoscopic findings, endoscopic ultrasound and computed tomography scan findings, type of surgery, histological analysis of surgical specimen and the treatments used.

Exclusion criteria were: genetic gastric cancer, history of gastric surgery for any reason, history of endoscopic resection for superficial tumor prior to surgery (endoscopic mucosal resection or submucosal dissection), gastro-esophageal junction cancer, non-adenocarcinomatous gastric tumor, adenocarcinoma infiltration of extra-gastric origin and absence of tumor residue on the pathology report. We also excluded the files with at least one major missing data (histological report of endoscopic biopsies or surgical specimen, digestive endoscopy report). The large number of excluded patients is due to the retrospective design of our study and the lack of computerization of medical data in some centers (incomplete paper records) and non-available histological report. Patients were treated in accordance with the Helsinki Declaration (World Medical Association Declaration of Helsinki. Ethical principles for medical research involving human subjects. Bulletin of the World Health Organization, 2001;79(4):373-374). All data were anonymously collected and, according to the Loi Jardé (French law amended by Order no. 2016-800 and its implementing decree no. 2016-1537 of 16/11/ 2016 relating to research involving the human person), no patient consent was needed, as the treatment implemented in this study was the standard recommended therapy.

To create the score, our study population was divided into two groups: GLP group and non-linitic adenocarcinoma (non-GLP or control group).

\section{Definition of GLP and pathological analysis}

The diagnosis of GLP was retained if the three following criteria were mentioned on the pathology report of surgical specimen:

Macroscopic examination of the surgical specimen showing segmental or pangastric diffuse parietal thickening.

Histological examination showing an abundant and diffuse fibrous stromal reaction extended throughout the gastric lining to the sub serosa.

Histological examination showing a carcinoma with more than $50 \%$ poorly cohesive cells having classical SRC morphology.

The pathology reports of gastrectomy were all reviewed for validation by an expert pathologist in the reference center of Assistance Publique des Hôpitaux de Paris (AP-HP) for the treatment of oesogastric tumors. In some doubtful cases, a re-reading of the glass slides was carried out. If one of the three criteria was absent, the patient was excluded from the GLP group even if the clinical presentation and the morphological assessment appeared compatible with the diagnosis. 
Patients in the non-GLP group were randomly selected from the same centers without matching regardless of the presence or not of signet ring cells on surgical specimen.

All pathology reports of gastrectomy have been standardized according to the latest UICC AJCC 2016 classification [15].

\section{Parameters for diagnostic score}

The parameters taken into account for the creation of the score were demographic characteristics, symptoms at the diagnosis of GA, description of the initial upper gastrointestinal endoscopy and if available of gastric endoscopic ultrasound, description of pre-therapeutic abdominal computed tomography scan and histological description of endoscopic biopsies at diagnosis (cf Tables 1 and 2). The biological parameters included for the creation of the score were: total blood count (anemia, increased neutrophils count, thrombocytosis), high neutrophil-to-lymphocyte ratio, elevated
C-reactive protein, hypoalbuminemia, increased tumor markers (carcinoembryonic antigen, carbohydrate antigen 19-9). Further details are provided in Supplementary Tables 1-5.

\section{Statistical analysis}

Patient characteristics are presented using medians and interquartile ranges for quantitative data and counts with percentages for qualitative data. The characteristics of patients with and without GLP were compared using Wilcoxon tests for quantitative data and $\mathrm{Chi}^{2}$ tests or Fisher tests for qualitative data.

There were $12.6 \%$ of missing data among all the predictors considered, and only $5 \%$ of patients had no missing data. Under the hypothesis of missingness at random, we used multiple imputations by chained equations to generate 20 imputed datasets. The diagnostic score was constructed with the most frequently selected predictors on
Table 1 General characteristics of the study population

\begin{tabular}{|c|c|c|c|c|c|}
\hline \multirow{2}{*}{$\begin{array}{l}\text { Variables } \\
\text { Gender, } n(\%)\end{array}$} & \multicolumn{2}{|c|}{ GLP $N=71$} & \multicolumn{2}{|c|}{ Non-GLP $N=149$} & \multirow[t]{2}{*}{$P$ value } \\
\hline & & & & & \\
\hline Female/male & $35 / 36$ & $(49 / 51)$ & $45 / 104$ & $(30 / 70)$ & 0.007 \\
\hline \multicolumn{6}{|l|}{ Age at diagnosis (years) } \\
\hline Median (IQR) & 57 & $(45.5-63)$ & 64 & $(56-71.5)$ & $<0.001$ \\
\hline \multicolumn{6}{|c|}{ Time to first symptoms-cancer diagnosis (days) } \\
\hline Median (IQR) & 103 & $(72-184)$ & 67 & $(17-181)$ & 0.02 \\
\hline \multicolumn{6}{|l|}{ Tumor stage at diagnosis, $\mathrm{n}(\%)$} \\
\hline Localized tumor & 57 & $(80)$ & 142 & $(95)$ & \\
\hline Metastatic tumor & 14 & $(20)$ & 7 & $(5)$ & \\
\hline \multicolumn{6}{|l|}{ Preoperative treatment } \\
\hline Upfront surgery & 24 & $(34)$ & 69 & $(46)$ & \\
\hline Systemic chemotherapy & 47 & $(66)$ & 80 & $(54)$ & \\
\hline Systemic chemotherapy + PIPAC $^{\mathrm{a}}, \mathrm{n}(\%)$ & 2 & (3) & 0 & $(0)$ & \\
\hline \multicolumn{6}{|l|}{ Clinical symptoms at diagnosis, $\mathrm{n}(\%)^{\mathrm{b}}$} \\
\hline Poor general status & 41 & $(58)$ & 39 & (26) & $<0.001$ \\
\hline Undernutrition & 32 & $(45)$ & 40 & $(27)$ & 0.002 \\
\hline Dysphagia & 14 & $(20)$ & 6 & (4) & $<0.001$ \\
\hline Epigastric pain & 58 & $(82)$ & 97 & $(65)$ & 0.01 \\
\hline Vomiting & 11 & $(15.5)$ & 14 & $(9.5)$ & 0.255 \\
\hline Digestive hemorrhage & 4 & $(5.5)$ & 25 & $(17)$ & 0.03 \\
\hline Occlusive syndrome & 7 & $(10)$ & 7 & $(5)$ & 0.151 \\
\hline Perforation & 3 & (4) & 4 & (3) & 0.684 \\
\hline \multicolumn{6}{|l|}{ Histological diagnosis, n (\%) } \\
\hline Unique upper digestive endoscopy & 49 & (69) & 140 & $(94)$ & $<0.001$ \\
\hline Repeated upper digestive endoscopies & 9 & $(12.5)$ & 7 & $(5)$ & $<0.001$ \\
\hline Upper endoscopic ultrasound & 2 & (3) & 1 & $(0.5)$ & \\
\hline Exploratory celioscopy & 7 & $(10)$ & 0 & $(0)$ & \\
\hline Inaugural surgery & 4 & (5.5) & 1 & $(0.5)$ & \\
\hline
\end{tabular}

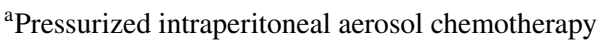

${ }^{\mathrm{b}}$ Several symptoms may be associated 
Table 2 Characteristics of endoscopic findings and imaging in the study population

\begin{tabular}{|c|c|c|c|c|c|}
\hline \multirow{2}{*}{$\begin{array}{l}\text { Variables } \\
\text { Upper gastrointestinal endoscopy, } n(\%)^{\mathrm{a}}\end{array}$} & \multicolumn{2}{|c|}{ GLP $N=71$} & \multicolumn{2}{|c|}{$\begin{array}{l}\text { Non-GLP } \\
N=149\end{array}$} & \multirow[t]{2}{*}{$P$ value } \\
\hline & & & & & \\
\hline Single ulcer & 27 & $(38)$ & 79 & $(53)$ & 0.04 \\
\hline Ulcerations or multiple erosions & 16 & $(22.5)$ & 10 & $(6.5)$ & 0.001 \\
\hline Ulcer-budding tumor & 4 & $(5.5)$ & 53 & $(35.5)$ & $<0.001$ \\
\hline Large gastric folds or thickening on one segment & 40 & $(56)$ & 9 & (6) & $<0.001$ \\
\hline Large gastric folds or diffuse thickening & 15 & $(21)$ & 0 & $(0)$ & $<0.001$ \\
\hline Difficulty of insufflation & 13 & $(18)$ & 0 & $(0)$ & $<0.001$ \\
\hline Stenosis & 21 & $(29.5)$ & 18 & (12) & 0.001 \\
\hline Pangastric tumor infiltration & 17 & $(24)$ & 0 & $(0)$ & $<0.001$ \\
\hline Tumor infiltration extending to the duodenal bulb & 8 & $(11)$ & 1 & (1) & $<0.001$ \\
\hline Tumor diagnosis not mentioned on the macroscopic aspect & 16 & $(22.5)$ & 4 & (3) & $<0.001$ \\
\hline \multicolumn{6}{|l|}{ Upper endoscopic ultrasound, $n(\%)^{\mathrm{a}}$} \\
\hline Circumferential thickening & 28 & $(39.5)$ & 8 & $(5.5)$ & $<0.001$ \\
\hline Pan gastric thickening & 13 & $(18)$ & 0 & $(0)$ & $<0.001$ \\
\hline Thickening of an entire segment or a limited part & 33 & $(46.5)$ & 77 & $(52)$ & 0.004 \\
\hline Wall thickening predominant on the 3rd hyperechoic layer & 15 & $(21)$ & 0 & $(0)$ & $<0.001$ \\
\hline Layer fusion & 14 & $(20)$ & 11 & $(7.5)$ & 0.008 \\
\hline Suspicious peri-gastric adenopathy & 26 & $(36)$ & 44 & $(29.5)$ & 0.585 \\
\hline \multicolumn{6}{|l|}{ Scanner, $n(\%)^{\mathrm{b}}$} \\
\hline Localized parietal abnormality & 43 & $(60.5)$ & 95 & (64) & 0.63 \\
\hline Diffuse parietal abnormality & 19 & $(27)$ & 4 & (3) & $<0.001$ \\
\hline Circumferential parietal abnormality & 33 & $(46.5)$ & 17 & $(11.5)$ & $<0.001$ \\
\hline Suspicious peri-gastric adenopathy & 21 & $(29.5)$ & 66 & (44) & 0.128 \\
\hline
\end{tabular}

${ }^{a}$ Several possible lesions in the same patient

${ }^{\mathrm{b}}$ Parietal abnormalities $=$ thickening \pm parietal enhancement or endoluminal bud these 20 datasets. To take into account for both the low variable/individual ratio with 71 patients having GLP and 49 candidate covariates and the high risks of collinearities between candidate covariates, we used a generalized linear model with LASSO regularization (with tenfold cross-validation to select $\lambda$ parameter) to build the multivariable model and select predictors. Finally, due to the near separation of some variables (no individuals in any of the modalities), a Firth penalized logistic regression was performed to assess the respective importance of each predictor. The Firth logistic model was applied to each of the 20 imputed datasets and the resulting mean value of each coefficient was used to construct the score. We rounded the coefficient to obtain an easy to calculate diagnostic score.

The performances of this score were assessed through discrimination measures: ROC curve, area under the curve (AUC), sensitivity and specificity at the chosen threshold.

Finally, bootstrap resampling (200 bootstrap resampling for each of the 20 imputed datasets) allowed us to obtain an internal validation to correct for overoptimism in the discrimination measures. All statistical analyses were performed using R software.
The methodology used is in agreement with the criteria defined by the TRIPOD checklist. Further details are provided in Supplementary Table S6.

\section{Results}

\section{Patients' characteristics}

The files of 457 patients aged over 18 years who underwent a gastrectomy for gastric cancer were reviewed. Among them, 72 records were not analyzable due to major missing data. In the remaining 385 patients, 165 presented an exclusion criteria. Therefore, a total of 220 patients (71 in the GLP group and 149 in the control group) met the inclusion criteria and were included in the analysis (Fig. 1).

The general characteristics of the study population at diagnosis are presented in Table 1. Several statistical differences regarding epidemiological data and clinical presentation at diagnosis were noted between GLP and non-GLP patients. In the GLP group, the population was younger $(p<0.001)$ with a higher proportion of women $(p=0.007)$, 
a longer diagnostic time $(p=0.02)$ and the need to repeat iterative biopsy endoscopy more frequently $(p<0.001)$. Clinical presentation also differed with a higher proportion of patients with general impairment $(p<0.001)$, undernutrition $(p=0.002)$, dysphagia $(p<0.001)$ and epigastralgia $(p=0.01)$. In the non-GLP group, we noted a higher proportion of patients explored for anemia or with externalized digestive hemorrhage $(p=0.03)$. Upfront surgery was performed in four patients in the GLP group (surgical decision at baseline: $n=3$, perforation: $n=1$ ) and one patient in the non-GLP group (perforation).

The description of the main additional examinations carried out for diagnostic purposes is presented in Table 2 .

\section{Endoscopic findings}

Several statistical differences were noted at endoscopic examination between GLP and non-GLP patients. In the GLP group, there were more patients with large folds or macroscopic tumor infiltration on at least one segment $(p<0.001)$ with a higher frequency of multiple ulcerations or erosions in the suspected area $(p=0.001)$. Difficulty with insufflation and the presence of stenosis were also more frequently observed $(p<0.001)$. In the non-GLP group, there was a higher proportion of patients with a single ulcer or ulcer-budding tumor $(p<0.001)$. On endoscopic ultrasound, there was more frequent thickening on at least one segment $(p<0.04)$ or pangastric thickening in the GLP group $(p<0.001)$. This one was more frequently circumferential with predominance over the third hyperechoic layer or fusion aspect of the layers $(p<0.001)$.

\section{Imaging}

On contrast-enhanced computed tomography scan (13\% with opacification with water or contrast medium), we observed a higher proportion of diffuse parietal involvement and circumferential thickening of the gastric wall.

\section{Biology}

Among all the biological parameters studied, only anemia was significantly more frequent in the non-GLP group. No significant differences were observed on the other characteristics of blood count and CRP, serum albumin and tumor marker elevation frequency (ACE and CA 19-9) between both groups.

\section{Pathological findings}

The comparative analysis of the histological characteristics of the gastrectomy specimens is presented in Table 3. Again, we noted several statistically significant differences between the two groups. In the GLP group, the number of total gastrectomy was higher with more incomplete resection. The disease was more frequently pangastric with an increased number of T4 status, positive lymph nodes, distant metastases and poorly cohesive and/or SRC contingent. Among the patients with positive lymph nodes, we observed more frequently an N3 status in the GLP group than in the non-GLP group ( $45 \%$ vs $18 \%$ ). Among the patients with metastatic location, peritoneal carcinomatosis only was known preoperatively in 19 patients (14 in the GLP group and 5 in the nonGLP group) and found peroperatively in 15 patients (10 in the GLP group and 5 in the non-GLP group). In five patients of the GLP group, the tumor was a mixed type $(n=4)$ or a majority mucinous type $(n=1)$, according to the WHO 2010 classification. Of note, HER2 status positivity was low and the proportion of patients with Helicobacter pylori infection was similar in both groups.

\section{Diagnostic score}

The diagnosis score for gastric LP is presented in Table 4 . Regarding first results, six variables were selected to create the score: three variables corresponding to upper gastrointestinal endoscopic characteristics, the presence of large folds and/or gastric thickening on at least one segment (1.5 points), pangastric infiltration (2 points) and presence of gastric stenosis (1 point); two variables corresponding to endoscopic ultrasound characteristics, a circumferential thickening on at least one segment ( 0.5 points) and predominance of the lesion on the third hyperechoic layer (1 point); and one variable on histological report on endoscopic biopsies, presence of poorly cohesive cells and/or signet ring cells (1.5 points).

The score performance was evaluated by ROC curve (Fig. 2), with an AUC of 0.967 [0.948-0.987], a sensitivity of 94\% [89.9-97.3] and a specificity of $88.7 \%$ [81.7-95.8] for a threshold of 2.75 points (observed performances on one of the 20 imputed datasets).

After internal bootstrap validation (resampling), the corrected AUC was 0.959.

\section{Discussion}

\section{GLP: a clearly different entity}

Firstly, our results confirmed that GLP tumor has to be considered as a different entity from non-GLP tumors with different epidemiological, clinical, radiological and histological presentation. The importance of the differences observed at diagnosis between these two types of gastric tumors makes it necessary to use a reliable tool that clearly differentiates them. 
Table 3 Histological characteristics of the gastrectomy specimens

\begin{tabular}{|c|c|c|c|c|c|}
\hline \multirow{2}{*}{$\begin{array}{l}\text { Variables } \\
\text { Type of gastrectomy, } n(\%)\end{array}$} & \multicolumn{2}{|c|}{ GLP $N=71$} & \multicolumn{2}{|c|}{ Non-GLP $N=149$} & \multirow[t]{2}{*}{$P$ value } \\
\hline & & & & & \\
\hline Total & 61 & $(86)$ & 86 & $(58)$ & $<0.001$ \\
\hline Partial & 10 & (14) & 63 & $(42)$ & $<0.001$ \\
\hline \multicolumn{6}{|l|}{ Resection, $n(\%)$} \\
\hline R0 & 46 & $(65)$ & 141 & (94) & $<0.001$ \\
\hline $\mathrm{R} 1$ & 25 & $(35)$ & 7 & $(5)$ & \\
\hline $\mathrm{R} 2$ & 0 & $(0)$ & 1 & (1) & \\
\hline Tumour site, $n(\%)$ & & & & & $<0.001$ \\
\hline Pangastric & 30 & $(42)$ & 0 & $(0)$ & \\
\hline Fundus & 12 & $(17)$ & 28 & (19) & \\
\hline Antrum/pylorus & 24 & (34) & 93 & $(62)$ & \\
\hline Antro-fundic junction or body & 5 & (7) & 28 & (19) & \\
\hline AJCC TNM stage, $n(\%)^{\mathrm{a}}$ & & & & & $<0.001$ \\
\hline pT1-T2 & 2 & (3) & 64 & $(43)$ & \\
\hline pT3-T4 & 69 & $(97)$ & 85 & $(57)$ & \\
\hline Positive lymph nodes (any N) & 55 & $(75.5)$ & 85 & $(57)$ & $<0.001$ \\
\hline M1 (metastatic location) & 24 & $(34)$ & 12 & (8) & $<0.001$ \\
\hline \multicolumn{6}{|l|}{ WHO classification, $n(\%)^{\mathrm{b}}$} \\
\hline Poorly cohesive (including signet ring cells) & 66 & $(93)$ & 33 & $(22)$ & \\
\hline Tubular & 0 & $(0)$ & 60 & $(40.5)$ & \\
\hline Papillary & 0 & $(0)$ & 6 & (4) & \\
\hline Mucinous & 1 & $(1.5)$ & 5 & $(3.5)$ & \\
\hline Mixed & 4 & $(5.5)$ & 21 & (14) & \\
\hline Unknown & 0 & $(0)$ & 24 & $(16)$ & \\
\hline \multicolumn{6}{|l|}{ Lymph node(s) } \\
\hline Number of lymph nodes analyzed, median (IQR) & 26 & $(18-34)$ & 22 & $(16-31)$ & 0.054 \\
\hline Number of invaded lymph nodes, median (IQR) & 8 & $(3-13)$ & 4 & $(3-9)$ & $<0.001$ \\
\hline \multicolumn{6}{|l|}{ HER $2, n(\%)^{\mathrm{c}}$} \\
\hline Positive & 1 & $(1.5)$ & 12 & $(8)$ & 0.112 \\
\hline Not determined & 16 & $(22.5)$ & 19 & (13) & \\
\hline
\end{tabular}

${ }^{\mathrm{a}}$ UICC/AJCC 2016

${ }^{\mathrm{b}}$ WHO classification 2010

${ }^{c}$ HER2 status has sometimes been determined on endoscopic biopsies

\section{A new diagnostic score for GLP}

To our knowledge, we are reporting the first diagnostic score to discriminate GLP from other GA. This score has an excellent diagnostic performance to predict the existence of GLP with an AUC of 0.967 , a sensitivity of $94 \%$ and a specificity of $88.7 \%$ for a threshold of 2.75 points. The resampling by bootstrap allowed us to obtain an internal validation of the score performances with a corrected AUC of 0.959 which reinforce its viability. The 2/1 ratio between the GLP and the non-GLP group and the absence of a priori selection of the control group allow a satisfactory validation sample to be obtained.

The six variables used to create the score include three endoscopic parameters: the presence of large folds and/or parietal thickening on at least one segment (1.5 points), pangastric infiltration ( 2 points) and the presence of gastric stenosis (1 point); two endoscopic ultrasound parameters: circumferential thickening on at least one segment (0.5 points) and thickening of the third hyperechoic layer (1 point); and a histological parameter: presence of poorly cohesive and/or SRC (1.5 points).

The GLP group was identified using three strict criteria based on histological analysis of the gastrectomy specimen which is considered as the gold standard for the positive diagnosis of GLP. In addition, this diagnosis was validated by a centralized review of histological reports by a pathologist from a center specializing in the management of esogastric tumors using a keyword grid and in some cases a re-reading of the glass slides. As LP is a rare entity, obtaining a group of 71 patients who were included using only the current gold standard can be considered 
Fig. 1 Flowchart

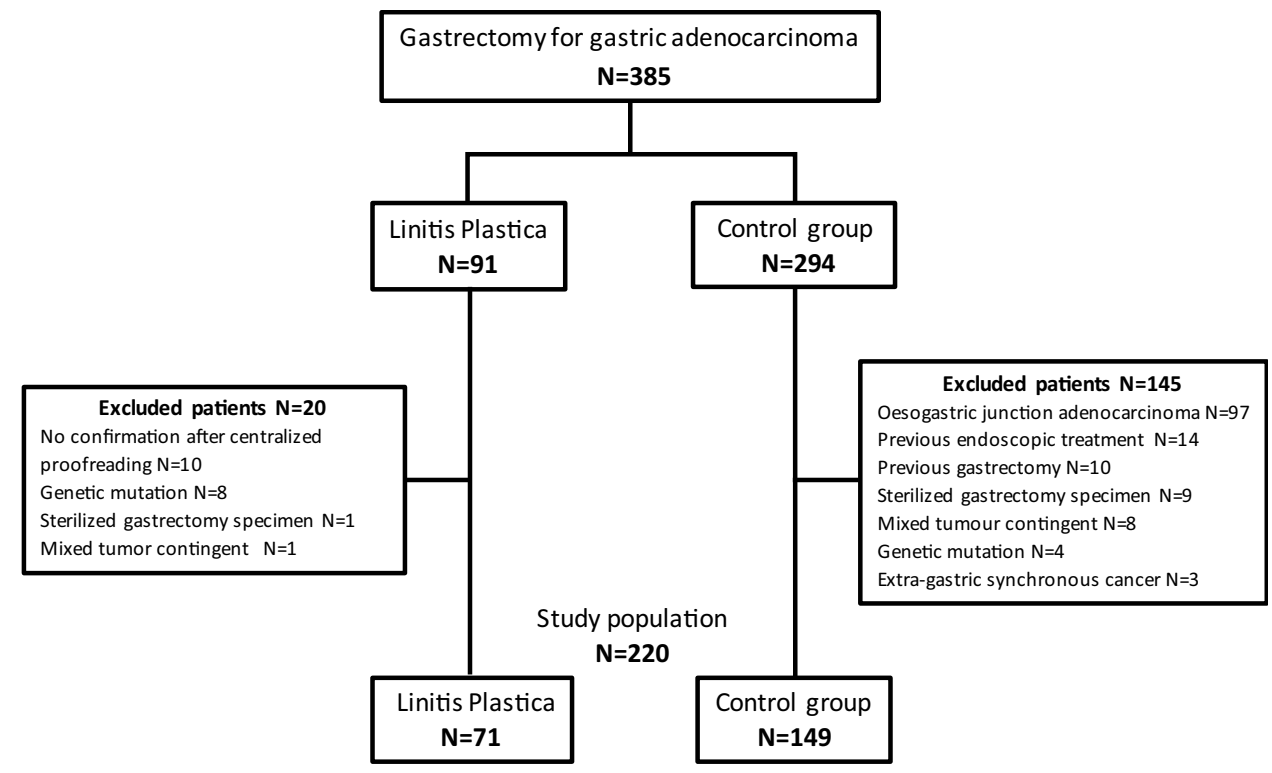

Table 4 Diagnostic score of gastric linitis plastica

\begin{tabular}{lll}
\hline Items & $\beta^{*}$ & Point \\
\hline $\begin{array}{l}\text { Upper gastrointestinal endoscopy } \\
\text { Large folds and/or gastric thickening on at least one }\end{array}$ & 3.18 & 1.5 \\
$\quad$ segment & 4.33 & 2 \\
Pangastric infiltration & 1.63 & 1 \\
Stenosis & & \\
Upper endoscopic ultrasound & 0.18 & 0.5 \\
Circumferential thickening & 2.49 & 1 \\
Thickening of the gastric wall predominant on the third & & \\
$\quad$ hyperechoic layer & & \\
Histology of gastric biopsies & 3.14 & 1.5 \\
Poorly cohesive and/or signet ring cells &
\end{tabular}

Each item scores 0 if absent or the tabulated value if present. The diagnostic score is the sum of each item and ranges from 0 to 7.5 ; a higher score indicates a higher probability of linitis plastic. The chosen threshold is 3: patients with a score $<3$ are considered not having gastric linitis plastica and patients with a score $\geq 3$ are considered having gastric linitis plastica

$* \beta$ Coefficients are obtained with Firth penalized logistic regression model

a large sample. Among the various parameters analyzed to create the score, many differed significantly between the two samples. The differences in clinical, endoscopic, scannographic and histological presentation observed in the GLP group have been previously reported in the literature underlining the quality of our sampling $[8,9$, 16]. The percentage of patients with SRC adenocarcinoma $(22 \%)$ in the control group was also in agreement with the literature [17-19].

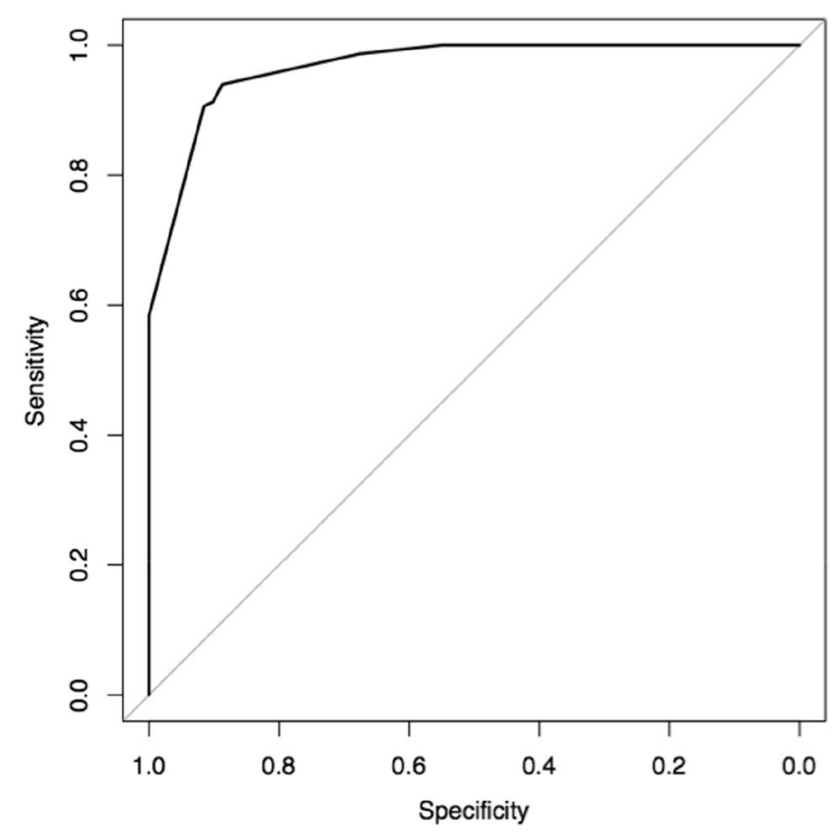

Fig. 2 ROC curve. The AUC is 0.967 [0.948-0.987]; using a threshold of 2.75 , the sensitivity is $94 \%$ [89.9-97.3] and the specificity is $88.7 \%$ [81.7-95.8]

\section{GLP: a lack of a consensual definition to date}

Histological analysis of the surgical specimen is not a tool that can be easily used in clinical practice given the high frequency of GLP that will never be operated on, mainly because of the greater aggressiveness of this pathology. Therefore, the definitions currently proposed are mainly based on upper gastrointestinal endoscopy. Thus, Pedrazzani et al. [20] defined GLP as a thickening and stiffening of the gastric wall which involve circumferentially at least 
one-third of the stomach, and according to Endo et al. [21] more than two-thirds of the stomach. More recently, Agnes et al. [9] proposed the following definition: thickening of the gastric wall, with lack of distensibility, which involves more than one-third of the gastric surface, both as a circumferential involvement of more than one area, or a semicircular involvement of more than two areas. Finally Jung et al. [16] proposed a decisional algorithm to diagnose GLP based on macroscopic and microscopic data of the initial upper gastrointestinal endoscopy. However, these definitions are not validated and the inter-observer reproducibility of the description of endoscopic lesions is not known.

GLP: a new diagnostic score that uses routine exams.

Endoscopic ultrasound is usually recommended for the diagnosis of GLP, but its diagnostic value in distinguishing it from classical GA has never been studied [22]. Endoscopic ultrasound puncture seems a useful tool in difficult cases, but remains poorly evaluated and most often useless [23]. Although the presence of poorly cohesive cells and/or SRC is almost constant in GLP, they can frequently be found in diffuse gastric adenocarcinomas and therefore do not constitute a discriminant parameter. Our score allows the diagnosis of GLP to be carried out with high sensitivity and specificity using usual explorations for the diagnosis of GA. Even if the inter-observer reproducibility of the different examinations is poorly known, the description of the macroscopic aspect on the upper gastrointestinal endoscopy, the analysis of the different gastric wall layers in endoscopic ultrasound and the histological description of gastric tumors represent routine procedures applicable in current practice to establish a diagnostic score. Despite some differences, no discriminating clinical parameters were found in the GLP sample. This is in agreement with the literature which reports that gastric cancer symptoms are non-specific and that in the event of a positive diagnosis there is no clinical sign to distinguish a particular tumor subtype. The CT scan has been recently shown to be a useful tool for the diagnosis of GLP [24]. This is confirmed by our data which show a significantly increased frequency of circumferential and/or pangastric parietal abnormalities. However, these two morphological parameters remain less discriminating than those observed on the upper gastrointestinal endoscopy and the endoscopic ultrasound.

\section{GLP diagnostic score: a new tool to standardize its management}

Despite the severity of this pathology, medico-surgical management of gastric LP remains poorly codified. The rarity of this gastric tumor, the absence of a consensual definition and the confusion created by the term signet ring cell carcinoma contribute to the absence of therapeutic advances, although the GLP has different characteristics and a poorer prognosis.
Other scores have already been validated in GA in patients treated, notably to establish survival-predictive factors after gastrectomy [25-27] or in metastatic patients undergoing chemotherapy $[28,29]$. The validation of a diagnostic score specific to GLP provides a new homogeneous pre-therapeutic definition that could standardize the management of this pathology, which is considered chemoresistant [30].

\section{Limitations}

Nevertheless, our study has some limitations. The data were collected retrospectively with a limited number of patients in the LP sample and no systematic centralized re-reading of all the glass slides. The retrospective design of the study led to some missing data. However, all the files including the pathology reports were centrally reviewed to reduce the number of missing data. Some inaccuracies in upper gastrointestinal endoscopy, endoscopic ultrasound and CT scan reports may also have led to misinterpretation of some data; however, this parameter has been taken into account in the creation of our score by performing multiple imputations using chain equations. Furthermore, our score was not externally validated in an independent cohort. Nevertheless, the rarity of this pathology and the difficulty of obtaining a homogeneous study group make it difficult to carry out such work.

\section{Conclusion}

We have constructed and validated the first score to diagnose GLP with high sensitivity and specificity (Saint Louis linitis score). This one is composed of six parameters easily applicable in clinical practice and allows to determine a homogeneous group of patients in a pathology where there is no consensual definition. The use of this score may help to improve the therapeutic management of this subtype of GA, in particular the interest of preoperative chemotherapy and the extent of gastric resection if planned. However, an external validation is necessary to integrate this new score into clinical practice.

\section{Compliance with ethical standards}

Conflict of interest All the authors declare no conflict of interest for this article (ICMJE Form for Disclosure of Potential Conflicts of Interest).

Ethical standards All procedures followed were in accordance with the ethical standards of the responsible committee on human experimentation (institutional and national) and with the Helsinki Declaration of 1964 and later versions. 
Informed consent All data were anonymously collected and, according to the Loi Jardé, no patient consent was needed, as the treatment implemented in this study was the standard recommended therapy.

\section{References}

1. Ferlay J, Soerjomataram I, Dikshit R, Eser S, Mathers C, Rebelo $\mathrm{M}$, et al. Cancer incidence and mortality worldwide: Sources, methods and major patterns in GLOBOCAN 2012. Int J Cancer. 2015;136(5):359-86.

2. Karimi P, Islami F, Anandasabapathy S, Freedman ND, Kamangar F. Gastric cancer: descriptive epidemiology, risk factors, screening, and prevention. Cancer Epidemiol Biomark Prev. 2014;23(5):700-13.

3. Lauren P. The two histological main types of gastric carcinoma: diffuse and so-called intestinal-type carcinoma An attempt at a histo-clinical classification. Acta Pathol Microbiol Scand. 1965;64:31-49.

4. Bosman FT, Carneiro F, Hruban RH, Theise ND (2010) World Health Organization (WHO) classification of tumours of the digestive system, 4th Edition. International Agency for Research on Cancer (IARC), Lyon, pp 45-79.

5. Japanese Gastric Cancer Association. Japanese classification of gastric carcinoma: 3rd English edition. Gastric Cancer. 2011;14(2):101-12.

6. Borrmann R. Geschwülste Des Magens und Des Duodenums, vol. 1. Berlin: Springer; 1926.

7. Siewert J, Stein H. Carcinoma of the gastroesophageal junctionclassification, pathology and extent of resection. Dis Esophagus. 1996;9:173-82.

8. Mastoraki A, Papanikolaou IS, Sakorafas G, Safioleas M. Facing the challenge of managing linitis plastic-review of the literature. Hepatogastroenterology. 2009;56(96):1773-8.

9. Agnes A, Estrella JS, Badgwell B. The significance of a nineteenth century definition in the era of genomics: linitis plastica. World J Surg Oncol. 2017;15(1).

10. Chang JM, Lara KA, Gray RJ, Pockaj BA, Wasif N. Clinical outcomes after surgery for linitis plastica of the stomach: analysis of a population cancer registry. Am Surg. 2017;83(1):23-9.

11. Blackham AU, Swords DS, Levine EA, Fino NF, Squires MH, Poultsides $\mathrm{G}$, et al. Is linitis plastica a contraindication for surgical resection: a multi-institution study of the US gastric cancer collaborative. Ann Surg Oncol. 2016;23(4):1203-11.

12. Mariette C, Carneiro F, Grabsch HI, van der Post RS, Allum W, de Manzoni G, et al. Consensus on the pathological definition and classification of poorly cohesive gastric carcinoma. Gastric Cancer Off J Int Gastric Cancer Assoc Jpn Gastric Cancer Assoc. 2018; $15: 123$.

13. Palli D, Bianchi S, Cipriani F, Duca P, Amorosi A, Avellini C, et al. Reproducibility of histologic classification of gastric cancer. Br J Cancer. 1991;63(5):765.

14. Flucke U, Mönig SP, Baldus SE, Zirbes TK, Bollschweiler E, Thiele J, et al. Differences between biopsy- or specimen-related Laurén and World Health Organization classification in gastric cancer. World J Surg. 2002;26(2):137-40.

15. Amin MB, Edge SB, Greene FL, et al., editors. AJCC cancer staging manual. 8th ed. New York: Springer; 2017.

16. Jung K, Park MI, Kim SE, Park SJ. Borrmann type 4 advanced gastric cancer: focus on the development of scirrhous gastric cancer. Clin Endosc. 2016;49(4):336-45.
17. Pernot S. Signet-ring cell carcinoma of the stomach: Impact on prognosis and specific therapeutic challenge. World J Gastroenterol. 2015;21(40): 11428.

18. Golembeski CP, Genta RM. Signet-ring cell carcinoma in gastric biopsies: expecting the unexpected. J Clin Pathol. 2013;66(2):136-9.

19. Piessen G, Amielh D, Messager M, Vinatier E, Leteurtre E, Triboulet JP, et al. Is pretreatment endoscopic biopsy a good predictor of signet ring cell histology in gastric carcinoma? World J Surg. 2012;36(2):346-54.

20. Pedrazzani C, Marrelli D, Pacelli F, Di Cosmo M, Mura G, Bettarini F, et al. Gastric linitis plastica: which role for surgical resection? Gastric Cancer. 2012;15(1):56-60.

21. Endo K, Sakurai M, Kusumoto E, Uehara H, Yamaguchi S, Tsutsumi N, et al. Biological significance of localized Type IV scirrhous gastric cancer. Oncol Lett. 2012;3(1):94-9.

22. Mocellin S, Pasquali S. Diagnostic accuracy of endoscopic ultrasonography (EUS) for the preoperative locoregional staging of primary gastric cancer. Cochrane Database Syst Rev. 2015;2:1-173.

23. Liu Y, Chen K, Yang X-J. Endoscopic ultrasound guided fine needle aspiration used in diagnosing gastric linitis plastica: metastatic lymph nodes can be valuable targets. J Gastroenterol Hepatol. 2018;34:202-6.

24. Morgant S, Artru P, Oudjit A, Lourenco N, Pasquer A, Walter T, et al. Computed tomography scan efficacy in staging gastric linitis plastica lesion: a retrospective multicentric French study. Cancer Manag Res. 2018;10:3825-31.

25. Han D-S, Suh Y-S, Kong S-H, Lee H-J, Choi Y, Aikou S, et al. Nomogram predicting long-term survival after d2 gastrectomy for gastric cancer. J Clin Oncol Off J Am Soc Clin Oncol. 2012;30(31):3834-40.

26. Hirabayashi S, Kosugi S, Isobe Y, Nashimoto A, Oda I, Hayashi $\mathrm{K}$, et al. Development and external validation of a nomogram for overall survival after curative resection in serosa-negative, locally advanced gastric cancer. Ann Oncol Off J Eur Soc Med Oncol. 2014;25(6):1179-84.

27. Zheng Z-F, Lu J, Wang W, Desiderio J, Li P, Xie J-W, et al. Development and external validation of a simplified nomogram predicting individual survival after R0 resection for gastric cancer: an international, multicenter study. Ann Surg Oncol. 2018;25:2383-90.

28. Narita Y, Kadowaki S, Oze I, Kito Y, Kawakami T, Machida N, et al. Establishment and validation of prognostic nomograms in first-line metastatic gastric cancer patients. J Gastrointest Oncol. 2018;9(1):52-63.

29. Kim SY, Yoon MJ, Park YI, Kim MJ, Nam B-H, Park SR. Nomograms predicting survival of patients with unresectable or metastatic gastric cancer who receive combination cytotoxic chemotherapy as first-line treatment. Gastric Cancer Off J Int Gastric Cancer Assoc Jpn Gastric Cancer Assoc. 2018;21(3):453-63.

30. Messager M, Lefevre JH, Pichot-Delahaye V, Souadka A, Piessen $\mathrm{G}$, Mariette C, et al. The impact of perioperative chemotherapy on survival in patients with gastric signet ring cell adenocarcinoma: a multicenter comparative study. Ann Surg. 2011;254(5):684-93.

Publisher's Note Springer Nature remains neutral with regard to jurisdictional claims in published maps and institutional affiliations. 